\title{
Anomalous BCS equation for a Luttinger superconductor
}

\author{
V.Mastropietro \\ Dipartimento di Matematica, Università "Tor Vergata" di Roma
}

September 17, 2017

\begin{abstract}
In the context of the Anderson theory of high $T_{c}$ cuprates, we develop a BCS theory for Luttinger liquids. If the Luttinger interaction is much stronger than the BCS potential we find that the BCS equation is quite modified compared to usual BCS equation for Fermi liquids. In particular $T_{c}$ predicted by the BCS equation for Luttinger liquids is quite higher than the usual $T_{c}$ for Fermi liquids.
\end{abstract}

\section{The anomalous BCS equation}

The equivalent of BCS theory for a Luttinger liquid has not formally worked out, despite the relevance of such theory for the problem of superconductivity in the high- $T_{c}$ cuprates, see [1] or the discussion in the following section. Such theory should describe superconductors which in their normal state are Luttinger liquids. In this paper we develop such theory using constructive quantum field theory techniques, applied in many other Luttinger liquid problems, see [2], [3]. In particular, we compute in a rigorous way the BCS self-consistence equation for a spinning Luttinger liquid (the Mattis model, see [4]) coupled with a BCS anomalous potential. We stress that it should be difficult to find similar results using different techniques: in fact the Mattis model plus a BCS interaction is not exactly solvable, and bosonization or conformal quantum field theory cannot be used to compute the correlation functions of theories with gap like the present one (see for instance [5]).

We will assume that an anomalous self energy has been introduced by some external influence in a Luttinger liquid

\footnotetext{
${ }^{1}$ See [1] pag.209
}

so that the model is described by the following hamiltonian

$$
H=H_{a}+H_{B C S}
$$

where $H_{a}$ is the Mattis model hamiltonian

$$
\begin{aligned}
& \frac{2 \pi}{L} \sum_{k, \omega, \sigma}\left(\omega k-p_{F}\right) \psi_{k, \omega, \sigma}^{+} \psi_{k, \omega, \sigma}^{-}-\lambda\left[\frac{2 \pi}{L}\right]^{3} \sum_{k_{1}, k_{2}, p} \\
& \sum_{\omega, \sigma, \sigma^{\prime}} v(p): \psi_{k_{1}, \omega, \sigma}^{+} \psi_{k_{1}-p, \omega, \sigma}^{-}:: \psi_{k_{2},-\omega, \sigma^{\prime}}^{+} \psi_{k_{2}+p,-\omega, \sigma^{\prime}}^{-}:
\end{aligned}
$$

where $\psi_{k, \omega, \sigma}^{ \pm}$are fermionic creation or annihilation operators with momentum? $k=2 n \pi / L,-L / 2 \leq n \leq \frac{L-1}{2}$, quasi-particle index $\omega= \pm 1$, spin $\sigma= \pm \frac{1}{2} ;|v(p)| \leq e^{-\kappa|p|}$. We assume that $\lambda>0$. The BCS interaction is described as usual, if $g>0$

$$
\begin{aligned}
& H_{B C S}=-\Delta \frac{2 \pi}{L} \sum_{k, \omega} \psi_{k, \omega, \frac{1}{2}}^{+} \psi_{-k,-\omega,-\frac{1}{2}}^{+} \\
& -\Delta \frac{2 \pi}{L} \sum_{k, \omega} \psi_{-k,-\omega,-\frac{1}{2}}^{-} \psi_{k, \omega, \frac{1}{2}}^{-}+\frac{\Delta^{2}}{g}
\end{aligned}
$$

We assume that $\Delta \in R$ and $\Delta \geq 0$. Note that if $\Delta=0$ the model is exactly solvable [4]; the system is a Luttinger liquid (it is perhaps the simplest spinning model showing Luttinger liquid behaviour) and shows spin-charge separation.

The ground state energy $E_{0}(\Delta)$ depends on $\Delta$; the BCS equation is the extremizing equation $\frac{\partial E_{0}(\Delta)}{\partial \Delta}=0$ which has the form (see [6] for the proof of a similar statement):

$$
\Delta=g \frac{2 \pi}{L} \sum_{k, \omega}<\psi_{k, \omega, \frac{1}{2}}^{+} \psi_{-k,-\omega,-\frac{1}{2}}^{+}>
$$

\footnotetext{
${ }^{2}$ We are considering the Mattis model on a lattice, while the original model introduced and solved in [4] is defined on the continuum
} 
If $\lambda=0$ the r.h.s of eq.(4) can be calculated very easily, as the hamiltonian can be put in diagonal form by performing a Bogolubov transformation. In the interacting case an exact solution is not available and we compute the r.h.s. of eq.(4) writing it as a convergent series for $\Delta, \lambda$ suitably small i.e. $\Delta, \lambda<\varepsilon$, if $\varepsilon$ is a suitable number $<<1$ (but no restriction is imposed on their ratio). Note that the restriction on the smallness of $\Delta, \lambda$ is done only for technical reasons in order to ensure convergence of the perturbative series. However it is very likely that our bound for the convergence radius are far from to be optimal, and instead our results hold also for $\lambda=O(1)$; if $\Delta=0$ we know that this is true directly from the exact solution [4] अ?.

Our main result is

There exists an $\varepsilon>0$ such that, for $\Delta, \lambda \leq \varepsilon$ the $B C S$ equation (4), in the limit $\beta \rightarrow \infty, L \rightarrow \infty$, can be written as

$$
\frac{a \Delta}{g}=\frac{\Delta}{\eta}\left[|\Delta|^{-\eta}-A^{-\eta}\right](1+\lambda f)
$$

where $\eta=\beta_{1} \lambda+O\left(\lambda^{2}\right)$ is a critical index, $f \equiv f(\lambda, \Delta)$, $|f| \leq$ const, and $A, a, \beta_{1}$ are positive constants.

Eq.(5) is difficult to solve in general; we study its non trivial solutions for two limiting cases, denoting by $\sigma(x)$ a function bounded by $|x|$ times a constant.

1) $\frac{\lambda}{g}<<1$, which means that the BCS hamiltonian is weakly perturbed by the Luttinger interaction. As one expects, the gap is given by

$$
\Delta=A e^{-\frac{a}{g}(1+\sigma(\lambda))}
$$

so that the gap is exponentially small and the Luttinger liquid behaviour of the system plays no role.

2) $\frac{\lambda}{g}>>1$, which means that the Mattis hamiltonian is weakly perturbed by the BCS interaction. We find, remembering that $\eta=\beta_{1} \lambda+O\left(\lambda^{2}\right)$

$$
\Delta=A\left[\frac{g}{a \eta}\right]^{\frac{1}{\eta}}\left[1+\sigma(\lambda)+\sigma\left(\frac{g}{\lambda}\right)\right]^{\frac{1}{\eta}}
$$

We see that the Luttinger interaction enhances strongly the gap with respect to the $\lambda=0$ case if $\frac{\lambda}{g}>>1$

$$
\Delta \simeq B e^{-\frac{a}{g}\left[\frac{g}{a \eta}\left|\log \frac{g}{a \eta}\right|\right]}>>B^{\prime} e^{-\frac{a}{g}}
$$

\footnotetext{
${ }^{3}$ The role of the lattice should play no role.
}

Note also the crucial role of the sign of the Luttinger interaction.

\section{A model for the high- $T_{c}$ cuprates}

In the previous section we have written a BCS equation for a Luttinger liquid and we have seen that, if the Luttinger interaction is much stronger than the BCS interaction, there is a large deviation from the Fermi liquid BCS equation and in particular the gap is much larger. Can this result be applied to the physics of high- $T_{c}$ cuprates?

The Anderson theory of high- $T_{c}$ cuprates is based on the following points:

1) the conduction electrons are confined on layers;

2)the interaction between electrons in the same layer is much stronger than the interlayer interaction;

3 ) the normal state of the electrons on the layers is a Luttinger liquid;

4) the interlayer pairing allows Cooper pairs to tunneling into an adiacent layer by the Josephson mechanism

The interlayer interaction is assumed to be given by the Josephson pair tunneling hamiltonian and some physical arguments for motivating this choice and for not considering single particle tunneling are given The model is then studied by the usual mean field BCS approximation?. However by point 3 ) one has to take into account in the resulting BCS equation the Luttinger liquid nature of the fermions in their normal state. This is a big problem as no theory showing the Luttinger liquid behaviour of bidimensional strongly interacting fermions exists so that the form of a Luttinger liquid BCS equation is essentially guessed by replacing in the usual BCS the Fermi propagator with the one dimensional Luttinger model propagator ${ }^{10}$.

As the above theory is a mean field theory, its results should be, as usual, independent from the dimensions; this

\footnotetext{
${ }^{4}$ See for instance pag.46-55 of [1]

${ }^{5}$ See also pag. 308 of $[1]$

${ }^{6}$ See for instance eq.(7) pag 321 of [1], or eq.(9) (in $d=2$ ) below

${ }^{7}$ See for instance the considerations based on the holon-spinon coherence at pag.50 of [1] or in the Clarke reprint [o]

${ }^{8}$ see eq.(16) pag.218 of [1] or the considerations after eq.(10) below

${ }^{9}$ In [1] this is essentially derived from experimental results

${ }^{10}$ See pag. 213 eq.(12) of [1]
} 
means that we can expect that coupling $d=1$ or $d=2$ Luttinger liquids and performing a BCS approximation on the pair tunnelling interaction, the results are qualitatively the same. In other words we expect that the predictions of a BCS theory for a superconductor whose normal state is a Luttinger liquid are qualitatively the same whatever its dimensions are. This is just what happens for the usual superconductors whose normal state is a Fermi liquid; the BCS approximation leads for instance to a gap or a critical temperature exponentially small in $g$ in any dimension. The crucial and very non trivial assumption is that there exist $d=2$ fermions with a Luttinger liquid behaviour in their normal state; but, if they exists, the results of a BCS theory of coupled Luttinger liquids should be essentially indipendent on their dimensions.

Considering one dimensional coupled Luttinger liquids, the BCS equation can computed in a rigorous way. The Luttinger liquids can be described by the Mattis model hamiltonian eq.(2), giving an extra chain index to the Fermi operators $i=a, b$. The two Mattis hamiltonians will be called $H_{a}$ and $H_{b}$. The pair-hopping hamiltonian $H_{\text {int }}$ is, following [1] [1]

$$
\begin{aligned}
& H_{i n t}=-g\left[\frac{2 \pi}{L} \sum_{k, \omega} \psi_{k, \omega, \frac{1}{2}, a}^{+} \psi_{-k,-\omega,-\frac{1}{2}, a}^{+}\right] \\
& {\left[\frac{2 \pi}{L} \sum_{k^{\prime}, \omega} \psi_{-k^{\prime},-\omega,-\frac{1}{2}, b}^{-} \psi_{k^{\prime}, \omega, \frac{1}{2}, b}^{-}\right]+\text {c.c. }}
\end{aligned}
$$

The total hamiltonian is $H_{a}+H_{b}+H_{\text {int }}$. Let we write in eq.(9)

$$
\frac{2 \pi}{L} \sum_{k, \omega} \psi_{k, \omega, \frac{1}{2}, i}^{+} \psi_{-k,-\omega,-\frac{1}{2}, i}^{+}
$$

as

$$
\begin{aligned}
& \frac{2 \pi}{L} \sum_{k, \omega}<\psi_{k, \omega, \frac{1}{2}, i}^{+} \psi_{-k,-\omega,-\frac{1}{2}, i}^{+}>+ \\
& \frac{2 \pi}{L} \sum_{k, \omega}\left[\psi_{k, \omega, \frac{1}{2}, i}^{+} \psi_{-k,-\omega,-\frac{1}{2}, i}^{+}-<\psi_{k, \omega, \frac{1}{2}, i}^{+} \psi_{-k,-\omega,-\frac{1}{2}, i}^{+}>\right]
\end{aligned}
$$

The BCS approximation consists in replacing $H_{\text {int }}$ with $H_{\text {int }}^{a p p}$ obtained neglecting the terms bilinear in the "fluctuations" i.e. in the second addend in eq. (10). We obtain

$$
H_{\text {int }}^{a p p}=-\Delta \frac{2 \pi}{L} \sum_{k, \omega} \psi_{k, \omega, \frac{1}{2}, a}^{+} \psi_{-k,-\omega,-\frac{1}{2}, a}^{+}
$$

\footnotetext{
${ }^{11}$ See eq.(7) pag.320

${ }^{12}$ See eq.(16) pag.218 of [1]
}

$$
-\Delta \frac{2 \pi}{L} \sum_{k, \omega} \psi_{-k,-\omega, \frac{-1}{2}, b}^{-} \psi_{k, \omega, \frac{1}{2}, b}^{-}+\frac{\Delta^{2}}{g}+\text { c.c. }
$$

and self-consistency requires eq.(值). Replacing $H_{\text {int }}$ with $H _ { \text { int } } ^ { a p p } \longdiv { 1 3 }$ has the effect that the model is described by two indipendent hamiltonians, $H=\tilde{H}_{a}+\tilde{H}_{b}$, each one given by eq.(1).

The BCS equation for coupled Luttinger liquids is then given by eq.(5). In the range of parameters physically reasonable (see point 2) above) i.e. if $\lambda>>g$ and noting that, as usual, $T_{c}$ is proportional to $\Delta$ (see below) we find that $T_{c} \simeq \tilde{B} e^{-\frac{a}{g}\left[\frac{g}{a \eta}\left|\log \frac{g}{a \eta}\right|\right]}$ i.e. much higher than $T_{c}$ for normal superconductor $T_{c} \simeq \tilde{B}^{\prime} e^{-\frac{a}{g}}$.

In order to compare our result with [1], note that the r.h.s. of eq.(4) computed ${ }^{\text {[4] }}$ in [1] is similar to our eq.(22) below (see especially the presence of the wave function renormalization) but $\sigma_{h} \simeq \Delta \gamma^{-\left(\beta_{1} \lambda+. .\right) h}$ is replaced by $\Delta$ i.e. the anomalous flow of the BCS gap is neglected. Such effect is on the other hand crucial for our analysis: all the conclusion drawn from eq.(5) in the preceding section are based on the fact that $\beta_{1} \neq 0$. In fact it is this anomalous enlarging of the gap due to the Luttinger interaction which produces a much larger solution of the BCS equation. On the other hand the gap renormalization in Luttinger liquids is a well known fact, see for instance the gap of the $X Y Z$ model, [9].

Finally we stress that one can try to study directly a model of coupled chains, see [7], without any approximation, and it could happen that the model is not really described by our BCS approximation. This has not real relevance for our analysis, as our aim was just to find a BCS equation for coupled system of fermions on planes with a Luttinger liquid behaviour, and we make a BCS computation in $d=1$ using the fact that a mean field theory like BCS should be insensitive to the dimensions. So it could be that our BCS equation eq.(5) could be applied to coupled planes and not chains, and on the other hand a detailed analysis of the model given of eq.(10) could give no insight on the problem of coupled planes as the behavior of the system depend on the dimensions. This is in fact just what happens in the usual BCS theory: the

\footnotetext{
13 The above computation follows the Anderson gap derivation, see chap. 7 in [1], but for semplicity we have neglected the electron phonon interaction, see eq.(21) pag. 219 of [1]

${ }^{14}$ see eq.(12) pag. 213
} 
BCS equation is qualitatively the same in any dimension, but in $d=1$ the mean field approximation is not correct. The real question is if really bidimensional Luttinger exists, but this question is not addressed here and we refer to $[1]$.

\section{Renormalization group analysis}

We discuss now how to compute the r.h.s. of eq.(4). We introduce as usual a set of Grassman variables $\psi_{\vec{k}, \omega, \sigma}^{ \pm}$, $\vec{k}=\left(k_{0}, k\right)$ and $k=\frac{2 \pi n_{1}}{L}, k_{0}=\frac{2 \pi\left(n_{0}+2^{-1}\right)}{\beta}$, if $n_{0}, n_{1}$ are integers and $-\frac{L}{2} \leq n_{1} \leq(L-1) / 2,-\frac{\beta}{2} \leq n_{2} \leq \frac{\beta-1}{2}$, if $1 / \beta$ is the temperature. The Grassmanian integration $P(d \psi)$ is defined by the anticommutative Wick rule with propagator

$$
g_{\omega}(\vec{k})=\frac{1}{-i k_{0}+\omega k-p_{F}}
$$

In general we denote by $\int\left\{\mathcal{D} \psi e^{-\int d \vec{k} \psi_{\vec{k}}^{+} h(\vec{k})^{-1} \psi_{\vec{k}}^{-}}\right\}$the Grassmanian integration with propagator $h(\vec{k})$, where $\int d \vec{k}=\frac{(2 \pi)^{2}}{L \beta} \sum_{\vec{k}} ;$ in particular

$$
P(d \psi)=\left\{\mathcal{D} \psi \prod_{\vec{k}, \omega, \sigma} e^{-\int d \vec{k} \psi_{\vec{k}, \omega, \sigma}^{+}\left(-i k_{0}+\omega k-p_{F}\right) \psi_{\vec{k}, \omega, \sigma}^{-}}\right\}
$$

Then we write the r.h.s. side of eq.(14) as a functional integral

$$
\begin{aligned}
& <\psi_{\vec{k}, 1, \frac{1}{2}}^{+} \psi_{-\vec{k},-1,-\frac{1}{2}}^{+}>= \\
& \frac{1}{\int P(d \psi) e^{-V(\psi)}} \int P(d \psi) e^{-V(\psi)} \psi_{\vec{k}, 1, \frac{1}{2}}^{+} \psi_{-\vec{k},-1,-\frac{1}{2}}^{+}
\end{aligned}
$$

where $V(\psi)=\lambda \bar{V}+\Delta P$ and

$$
\begin{gathered}
P=\sum_{\omega} \int d \vec{k}\left(\psi_{\vec{k}, \omega, \frac{1}{2}}^{+} \psi_{-\vec{k},-\omega,-\frac{1}{2}}^{+}+\psi_{-\vec{k},-\omega, \frac{-1}{2}}^{-} \psi_{\vec{k}, \omega, \frac{1}{2}}^{-}\right) \\
V=\int \prod_{i=1}^{4} d \vec{k}_{i} \delta\left(\sum_{i=1}^{4} \varepsilon_{i} \vec{k}_{i}\right) \\
\sum_{\omega, \sigma, \sigma^{\prime}}: \psi_{\vec{k}_{1}, \omega, \sigma}^{+} \psi_{\vec{k}_{2}, \omega, \sigma}^{-}:: \psi_{\vec{k}_{3},-\omega, \sigma^{\prime}}^{+} \psi_{\vec{k}_{4},-\omega, \sigma^{\prime}}^{-}:
\end{gathered}
$$

We evaluate the above Grassman integral using Wilsonian renormalization group techniques. It is convenient to write $k=k^{\prime}+\omega p_{F}$, where $k^{\prime}$ is the momentum measured from the Fermi surface.
We decompose the integration $P(d \psi)$ into a product of independent integrations. This can be done writing

$$
g_{\omega}\left(\vec{k}^{\prime}+\omega p_{F}\right)=\sum_{h=0}^{-\infty} g_{\omega}^{h}\left(\vec{k}^{\prime}+\omega p_{F}\right)+g_{\omega}^{1}\left(\vec{k}^{\prime}+\omega p_{F}\right)
$$

with

$$
g_{\omega}^{h}\left(\vec{k}^{\prime}+\omega p_{F}\right)=\frac{f_{h}\left(\vec{k}^{\prime}\right)}{-i k_{0}+\omega k^{\prime}}
$$

and $f_{1}\left(\vec{k}^{\prime}\right)=1-\chi\left(\vec{k}^{\prime}\right), \chi\left(\vec{k}^{\prime}\right) \equiv \bar{\chi}\left(\left|\vec{k}^{\prime}\right|\right)$ is a smooth compact support function such that $\chi\left(\vec{k}^{\prime}\right)=1$ for $\left|\vec{k}^{\prime}\right| \leq \gamma^{-1}$ and $\chi\left(\vec{k}^{\prime}\right)=0$ for $\left|\vec{k}^{\prime}\right| \geq 1$, if $\gamma>1$; moreover for $h \leq 0$

$$
f_{h}\left(\vec{k}^{\prime}\right)=\chi\left(\gamma^{-h} \vec{k}^{\prime}\right)-\chi\left(\gamma^{-h+1} \vec{k}^{\prime}\right)
$$

is a smooth compact support function non vanishing only for $\gamma^{h-2} \leq\left|\vec{k}^{\prime}\right| \leq \gamma^{h}$. Then $g_{\omega}^{1}\left(\vec{k}^{\prime}+\omega p_{F}\right)$ is the ultraviolet part of the propagator, while $\sum_{k=0}^{-\infty} g_{\omega}^{h}\left(\vec{k}^{\prime}\right)$ is the infrared part. Note that, from the compact support properties of $g_{\omega}^{h}$, the sum in eq.(12) is from 0 to $h_{\beta}$, where $\gamma^{h_{\beta}}=\frac{\pi}{\beta}$, as $\left|k_{0}\right| \geq \frac{\pi}{\beta}$. Let be $C_{h}^{-1}=\sum_{k=h_{\beta}}^{h} f_{k}$.

The ultraviolet integration is somehow special (and essentially trivial for the presence of the lattice) and we will not discuss it here, see [2]. If $\lambda=0$ the infrared integration can be done by performing the well known Bogolubov transformation to diagonalize the BCS hamiltonian. If $\lambda \neq 0$ the BCS gap and the wave function renormalization have a non trivial RG flow so that we have to perform a different Bogolubov transformation at each iteration of the RG. We set $Z_{0}=1$ : once the fields $\psi^{(0)}, \ldots, \psi^{(h+1)}$ have been integrated we have:

$$
\begin{aligned}
& \int\left\{\mathcal{D} \psi^{(\leq h)} \prod_{\omega= \pm 1} e^{-\int d \vec{k}^{\prime} C_{h} Z_{h} \vec{\psi}_{\vec{k}^{\prime}, \omega}^{(\leq h)+} \mathcal{G}_{\omega}^{(h)}\left(\vec{k}^{\prime}\right)^{-1} \vec{\psi}_{\vec{k}^{\prime}, \omega}^{(\leq h)-}}\right\} \\
& e^{-V^{h}\left(\sqrt{Z_{h}} \psi^{(\leq h)}\right)}
\end{aligned}
$$

if

$$
\vec{\psi}_{\vec{k}^{\prime}, \omega}^{(\leq h) \pm}=\left(\psi_{\vec{k}^{\prime}+\omega p_{F}, \omega, \frac{1}{2}}^{(\leq h) \pm}, \psi_{-\vec{k}^{\prime}-\omega p_{F},-\omega,-\frac{1}{2}}^{(\leq h) \mp}\right)
$$

and $\mathcal{G}^{(h)}\left(\vec{k}^{\prime}\right)^{-1}$ is defined by

$$
\left(\begin{array}{cc}
-i k_{0}+\omega k^{\prime} & \sigma_{h}\left(k^{\prime}\right) \\
\sigma_{h}\left(k^{\prime}\right) & -i k_{0}-\omega k^{\prime}
\end{array}\right)
$$

$V^{h}$ is called the effective potential at scale $\mathrm{h}$ and is given by

$$
V^{h}\left(\psi^{(\leq h)}\right)=\sum_{n=2}^{\infty} \int \prod_{i=1}^{n} d \vec{k}_{i}
$$




$$
W_{n}^{h}\left(\vec{k}_{1}, \ldots, \vec{k}_{n}\right) \prod_{i=1}^{n} \psi_{\vec{k}_{i}, \omega_{i}, \sigma_{i}}^{(\leq h) \varepsilon_{i}} \delta\left(\sum_{i=1}^{n} \varepsilon_{i}\left(\vec{k}_{i}^{\prime}+\omega_{i} p_{F}\right)\right)
$$

We define a localization operator $\mathcal{L}$ extracting the relevant or marginal part of the effective potential $V^{h}$ :

i) If $n>4$ then $\mathcal{L} W_{n}^{h}=0$;

ii) Let be $n=4$. In this case $\mathcal{L} W_{4}^{h}=0$ unless $\sum_{i=1}^{4} \varepsilon_{i} \omega_{i} p_{F}=0, \sum_{i=1}^{4} \varepsilon_{i}=0$ in which case the action is non trivial and it is given by

$$
\mathcal{L} W_{4}^{h}\left(\vec{k}_{1}^{\prime}+\omega_{1} p_{F}, \ldots .\right)=W_{4}^{h}\left(\omega_{1} p_{F}, \ldots\right)
$$

iii) if $n=2$ then if $\sum_{i} \varepsilon_{i}=0$

$$
\begin{aligned}
& \mathcal{L} W_{2}^{h}\left(\vec{k}_{1}^{\prime}+\omega_{1} p_{F}, \vec{k}_{2}^{\prime}+\omega_{2} p_{F}\right)=\left[W_{2}^{h}\left(\omega_{1} p_{F}, \omega_{2} p_{F}\right)+\right. \\
& \omega_{1} E\left(k^{\prime}+\omega_{1} p_{F}\right) \partial_{k} W_{2}^{h}\left(\omega_{1} p_{F}, \omega_{2} p_{F}\right)+k^{0} \\
& \left.\partial_{k_{0}} W_{2}^{h}\left(\omega_{1} p_{F}, \omega_{2} p_{F}\right)\right]
\end{aligned}
$$

while if $\sum_{i} \varepsilon_{i} \neq 0$ then

$$
\mathcal{L} W_{2}^{h}\left(\vec{k}_{1}^{\prime}+\omega_{1} p_{F}, \vec{k}_{2}^{\prime}+\omega_{2} p_{F}\right)=W_{2}^{h}\left(\omega_{1} p_{F}, \omega_{2} p_{F}\right)
$$

We can write then the relevant part of the effective potential as:

$\mathcal{L} V^{h}=\gamma^{h} n_{h} F_{\nu}^{h}+s_{h} F_{\sigma}^{h}+z_{h} F_{\zeta}^{h}+a_{h} F_{\alpha}^{h}+g_{2, h} F_{2}^{h}+g_{4, h} F_{4}^{h}$

where

$$
\begin{aligned}
& F_{i}^{h}=\sum_{\omega} \int d \vec{k}^{\prime} f_{i} \psi_{\vec{k}^{\prime}+\omega p_{F}, \omega, \sigma}^{(\leq h)+} \psi_{\vec{k}^{\prime}+\omega p_{F}, \omega, \sigma}^{(\leq h)-} \\
& F_{\sigma}^{h}=\sum_{\omega} \int d \vec{k}^{\prime} \psi_{\vec{k}^{\prime}+\omega p_{F}, \omega, \frac{1}{2}}^{(\leq h)+} \psi_{-\vec{k}^{\prime}-\omega p_{F},-\omega,-\frac{1}{2}}^{(\leq h)+}+\text { c.c. } \\
& F_{2}^{h}=\int\left[\prod_{i=1}^{4} d \vec{k}_{i} \delta\left(\sum_{i} \vec{k}_{i}\right) \sum_{\omega, \sigma, \sigma^{\prime}}\right. \\
& {\left[\psi_{\vec{k}_{1}^{\prime}+\omega p_{F}, \omega, \sigma}^{(\leq h)+} \psi_{\vec{k}_{2}^{\prime}+\omega p_{F}, \omega, \sigma}^{(\leq h),-}\right]\left[\psi_{\vec{k}_{3}^{\prime}-\omega p_{F},-\omega, \sigma^{\prime}}^{(\leq h),+} \psi_{\vec{k}_{4}^{\prime}-\omega p_{F},-\omega, \sigma^{\prime}}^{(\leq h),}\right]} \\
& F_{4}^{h}=\int\left[\prod_{i=1}^{4} d \vec{k}_{i} \delta\left(\sum_{i} \vec{k}_{i}\right) \sum_{\omega, \sigma, \sigma^{\prime}}\right. \\
& {\left[\psi_{\vec{k}_{1}^{\prime}+\omega p_{F}, \omega, \sigma}^{(\leq h)+} \psi_{\vec{k}_{2}^{\prime}+\omega p_{F}, \omega, \sigma}^{(\leq h),}\right]\left[\psi_{\vec{k}_{3}^{\prime}+\omega p_{F}, \omega, \sigma^{\prime}}^{(\leq h),+} \psi_{\vec{k}_{4}^{\prime}+\omega p_{F}, \omega, \sigma^{\prime}}^{(\leq h),}\right]}
\end{aligned}
$$

where $i=\nu, \alpha, \zeta, f_{\nu}=1, f_{\alpha}=\omega k^{\prime}, f_{\zeta}=-i k_{0}$. Moreover $g_{2,0}=\hat{v}(0) \lambda+O\left(\lambda^{2}\right),\left|g_{4,0}\right| \leq C \lambda^{2}, s_{0}=\Delta+O(\Delta \lambda)$, $a_{0}, z_{0}=O(\lambda), n_{0}=O(\Delta \lambda)$. We write eq.(13) as:

$$
\begin{aligned}
& \int \mathcal{D} \psi^{(\leq h)} e^{-\int d k^{\prime} \vec{\psi}_{k^{\prime}}^{(\leq h)+} C_{h} Z_{h-1}\left(k^{\prime}\right) \mathcal{G}^{(h-1)}\left(k^{\prime}\right)^{-1} \vec{\psi}_{k^{\prime}}^{(\leq h)-}} \\
& \left.e^{-\tilde{V}^{h}\left(\sqrt{Z_{h}} \psi^{(\leq h)}\right)}\right]
\end{aligned}
$$

where $\mathcal{G}^{(h-1)}\left(\vec{k}^{\prime}\right)^{-1}$ is defined as in eq.(13), with $h-1$ istead of $h, \tilde{V}^{h}=\mathcal{L} \tilde{V}^{h}+(1-\mathcal{L}) V^{h}$,

$$
\mathcal{L} \tilde{V}^{h}=\gamma^{h} n_{h} F_{\nu}^{h}+\left(a_{h}-z_{h}\right) F_{\alpha}^{h}+g_{2, h} F_{2}^{h}+g_{4, h} F_{4}^{h}
$$

and

$$
\begin{gathered}
Z_{h-1}\left(k^{\prime}\right)=Z_{h}+C_{h}^{-1} Z_{h} z_{h} \\
Z_{h-1}\left(k^{\prime}\right) \sigma_{h-1}\left(k^{\prime}\right)=Z_{h} \sigma_{h}\left(k^{\prime}\right)+Z_{h} C_{h}^{-1} s_{h}
\end{gathered}
$$

This means that we extract from the effective potential the terms leading to a mass and wave function renormalization. Now one can perform the integration respect to $\psi^{(h)}$, rescaling the effective potential $\hat{V}^{h}(\psi)=\tilde{V}^{h}\left(\sqrt{\frac{Z_{h}}{Z_{h-1}}} \psi\right)$ and

$$
\mathcal{L} \hat{V}^{h}=\gamma^{h} \nu_{h} F_{\nu}^{h}+\delta_{h} F_{\alpha}^{h}+\lambda_{2, h} F_{2}^{h}+\lambda_{4, h} F_{4}^{h}
$$

with $\nu_{h}=\frac{Z_{h}}{Z_{h-1}} n_{h}, \delta_{h}=\frac{Z_{h}}{Z_{h-1}}\left(a_{h}-z_{h}\right), \lambda_{i, h}=\left(\frac{Z_{h}}{Z_{h-1}}\right)^{2} g_{i, h}$ and $\vec{v}_{k}=\nu_{h}, \delta_{h}, \lambda_{h}$. We can rewrite eq.(18) as

$$
\begin{aligned}
& \int \mathcal{D} \psi^{(\leq h-1)} e^{-\int d k^{\prime} \vec{\psi}_{k^{\prime}}^{(\leq h)+} C_{h} Z_{h-1}\left(k^{\prime}\right) \mathcal{G}^{(h-1)}\left(k^{\prime}\right)^{-1} \vec{\psi}_{k^{\prime}}^{(\leq h)-}} \\
& \int \mathcal{D} \psi^{(h)} e^{-\int d k^{\prime} \vec{\psi}_{k^{\prime}}^{(h)+} \tilde{f}_{h}^{-1} Z_{h-1}\left(k^{\prime}\right) \mathcal{G}^{(h-1)}\left(k^{\prime}\right)^{-1} \vec{\psi}_{k^{\prime}}^{(h)-}} \\
& \left.e^{-\hat{V}^{h}\left(\sqrt{Z_{h-1}} \psi^{(\leq h)}\right)}\right]
\end{aligned}
$$

and the integration of $\psi^{(h)}$ has propagator

$$
g_{\omega, \omega^{\prime}}^{h}(x-y)=\frac{1}{Z_{h-1}} \int d \vec{k}^{\prime} e^{i \vec{k}^{\prime}(x-y)} \tilde{f}_{h}\left(\vec{k}^{\prime}\right) \mathcal{G}^{(h-1)}\left(\vec{k}^{\prime}\right)_{\omega, \omega^{\prime}}
$$

with $\mathcal{G}^{(h-1)}\left(\vec{k}^{\prime}\right)$ given by

$$
\begin{gathered}
\frac{1}{A_{h}}\left(\begin{array}{cc}
\left(-i k_{0}+\omega k^{\prime}\right) & -\sigma_{h-1}\left(k^{\prime}\right) \\
-\sigma_{h-1}\left(k^{\prime}\right) & \left.\left(-i k_{0}-\omega k^{\prime}\right)\right)
\end{array}\right) \\
A_{h}=-k_{0}^{2}-\vec{k}^{\prime 2}-\sigma_{h-1}\left(k^{\prime}\right)^{2}
\end{gathered}
$$

and $Z_{h-1} \equiv Z_{h-1}(0)$ and

$$
\tilde{f}_{h}=Z_{h-1}\left[\frac{C_{h}^{-1}}{Z_{h-1}\left(k^{\prime}\right)}-\frac{C_{h-1}^{-1}}{Z_{h-1}}\right]
$$

The result of this integration is in the same form as eq.(13) with $h$ replaced by $h-1$, and we can iterate.

Let we explain the main motivations of the integration procedure discussed above. In a renormalization group approach one has to identify the relevant, marginal and irrelevant interactions. By a power counting argument 
one sees that the terms bilinear in the fields are relevant and the quartic terms (or the bilinear ones with a derivative respect to some coordinate acting on the fields) are marginal. However there are too many kinds of marginal terms, depending on the labels $\omega_{i}$ and $\varepsilon_{i}$ on each fields, so that their renormalization group flow seems impossible to study. However (see [2], [3] for a similar procedure) the power counting can be improved and many marginal terms are indeed irrelevant; in particular all the marginal terms with four or two fields with $\sum_{i} \varepsilon_{i} \neq 0$ are indeed irrelevant. The reason is that such terms are generated contracting at least a non diagonal propagator and such propagators are smaller than the diagonal ones by a factor $\sigma_{h} \gamma^{-h}$, see eq.(19) below; this will be sufficient for improving the power counting, (see the last paper in [3] for the proof of a similar statement in the XYZ chain). Moreover also the marginal terms with $\sum_{i} \varepsilon_{i} \omega_{i} p_{F} \neq 0$ are irrelevant, by momentum conservation considerations. In fact if $\sum_{i} \varepsilon_{i} \omega_{i} p_{F} \neq 0$ then the momenta of the fermions cannot be all close to the Fermi surface; mathematically this means that, for the compact support properties of the propagators, there is an $\bar{h}$ such that all scattering process involving fermions such that $\sum_{i} \varepsilon_{i} \omega_{i} p_{F} \neq 0$ with scale $h \leq \bar{h}$ are vanishing.

The relevant terms are of two kinds; the $\nu$ terms, reflecting the renormalization of the Fermi momentum, and the $\sigma$ terms, related to the presence of a gap in the spectrum. The presence of the $\nu$ terms is due to the renormalization of the chemical potential, and in general one introduces a counterterm in the hamiltonian to fix the Fermi momentum, see [2]. In this case however there is no necessity of adding this counterterm; roughly speaking, $\mu$ can vary in the gap whithout changing the Fermi momentum i.e. the position of the singularity of the propagator. This is a $\mathrm{cru}$ cial point: if one had to put a $\Delta$-dependent counterterm in the hamiltonian, then considering $\frac{\partial E_{0}(\Delta)}{\partial \Delta}$ one would be forced to derive also such counterterm, and a much more complex BCS equation would appear. Regarding the other relevant term, they are related to the BCS gap generation. However due to the interaction the BCS gap has a non trivial flow, so that one has to perform different Bogolubov transformations at each integration.

Regarding the marginal terms, there is an anomalous wave function renormalization which one has to take into account, what is expected as if $\Delta=0$ the theory is a Luttinger liquid. In general the flow of the marginal terms can be controlled using some cancellations due to the fact that the Beta function is "close" (for small $u$ ) to the Mattis model Beta function. In eq. (20) we write the propagator as the Mattis model propagator plus a remainder, so that the Beta function is equal to the Mattis model Beta function plus a "remainder" which is small if $\sigma_{h} \gamma^{-h}$ is small.

Let be $h^{*}=\inf _{\mathrm{h}}\left\{\gamma^{h} \geq\left|\sigma_{h}\right|\right\}$. Note that, if $h^{*}$ is finite uniformly in $L, \beta$ so that $\left|\sigma_{h^{*}-1}\right| \gamma^{-h^{*}+1} \geq 1$ one has

$$
\left|g^{<h^{*}}(\vec{x})\right| \leq \frac{1}{Z_{h^{*}}} \frac{C_{M} \gamma^{h^{*}}}{1+\left(\gamma^{h^{*}}|\vec{x}|\right)^{M}}
$$

Moreover if $h \geq h^{*}$ we have

$$
\left|g_{\omega, \omega}^{h}(\vec{x})\right| \leq \frac{1}{Z_{h}} \frac{C_{M} \gamma^{h}}{1+\left(\gamma^{h}|\vec{x}|\right)^{M}}
$$

and

$$
\left|g_{\omega,-\omega}^{h}(\vec{x})\right| \leq \frac{1}{Z_{h}} \frac{\left|\sigma_{h}\right|}{\gamma^{h}} \frac{C_{M} \gamma^{h}}{1+\left(\gamma^{h}|\vec{x}|\right)^{M}}
$$

Moreover for $h \geq h^{*}$ the bound for the non diagonal propagator has a factor more $\frac{\left|\sigma_{h}\right|}{\gamma^{h}}$ with respect to the diagonal propagator. This is the reason for which the quartic terms with $\sum_{i} \varepsilon_{i} \neq 0$ are irrelevant, despite dimensionally marginal. Finally

$$
g_{\omega, \omega}^{h}(x-y)=g_{\omega, L}^{h}(x-y)+C_{2, \omega}^{h}(x-y)
$$

with

$$
g_{\omega, L}^{h}(x-y)=\int d \vec{k}^{\prime} \frac{e^{i \vec{k}^{\prime} x}}{Z_{h}} \frac{f_{h}\left(\vec{k}^{\prime}\right)}{-i k_{0}+\omega k^{\prime}}
$$

which is just the propagator "at scale h" of the Mattis model, and the other term verify the bound of $g_{\omega, \omega}^{h}(\vec{x} ; \vec{y})$ with an extra factor $\frac{\left|\sigma_{h}\right|}{\gamma^{h}}$.

We see from the above bounds that the propagator of the integration of all the scale between $h^{*}$ and $h_{\beta}{ }^{[5}$ has the same bound as the propagator of the integration of a single scale greater than $h^{*}$; this will be used to perform the integration of all the scales $<h^{*}$ in a single step, i.e. integrating directly $\psi^{\left(<h^{*}\right)}$. In fact $\gamma^{h^{*}}$ is a momentum scale and, roughly speaking, for momenta bigger than $\gamma^{h^{*}}$ the theory is "essentially" a massless theory (up to $O\left(\sigma_{h} \gamma^{-h}\right)$ terms) while for momenta smaller than $\gamma^{h^{*}}$ is a "massive" theory with mass $O\left(\gamma^{h^{*}}\right)$.

${ }^{15}$ Of course if $h^{*} \leq h_{\beta}$ there is no such integration. 
One can prove that the effective potentials $V^{h}$ are well defined, if the running coupling constants are small enough. More precisely, let we write eq.(14) in coordinate space and let be $\tilde{W}_{n}^{k}$ the corresponding kernel; it holds that

Lemma:Assume that $h^{*}$ is is finite uniformly in $L, \beta$ and that for any $h>k \geq h^{*}$ there exists an $\varepsilon$ such that $\left|\vec{v}_{h}\right| \leq \varepsilon$ and $\left|\frac{\sigma_{h+1}}{\sigma_{h}}\right| \leq \gamma^{c_{a} \varepsilon},\left|\frac{Z_{h+1}}{Z_{h}}\right| \leq \gamma^{c_{b} \varepsilon^{2}}$ with $c_{a}, c_{b}$ postive constants. Then there exist a constant $C$ such that

$$
\left\|\tilde{W}_{n}^{k}\right\| \leq N \beta \varepsilon C \gamma^{-k\left(\frac{n}{2}-2\right)}
$$

The proof of the above lemma is an immediate modification of ones existing in literature, see in particular [3].

In order to prove that the effective potentials are well defined we have to show that the above conditions of smallness in the above lemma on the running coupling constants are verified.

The beta function for $\nu_{h}$ is

$$
\nu_{h-1}=\gamma \nu_{h}+\beta_{h}+\tilde{\beta}_{h}
$$

where $\beta_{h}$ is the contribution to $\nu_{h}$ obtained setting $\sigma_{k^{\prime}}=$ $0,0 \leq k^{\prime} \leq h$, so it is exactly equal to 0 by the parity properties of the Mattis model, and

$$
\left|\tilde{\beta}_{h}\right| \leq C \frac{\sigma_{h}}{\gamma^{h}} \lambda^{2}
$$

is the remaining part. Iterating the above relation we find

$$
\nu_{h-1}=\gamma^{|h|} \sum_{k=0}^{h} \gamma^{k} \tilde{\beta}_{k} \leq \gamma^{\left|h^{*}\right|} \lambda^{2} \sum_{k}\left|\sigma_{k}\right| \leq C|\lambda|
$$

as $\sigma_{k} \simeq \Delta \gamma^{\eta_{1} k}, \eta_{1}=-\beta_{1} \lambda+O\left(\lambda^{2}\right)$, see below.

The Beta function can be written, for $0 \geq h \geq h^{*}$. :

$$
\begin{aligned}
& \lambda_{2, h-1}=\lambda_{h}+G_{2}^{1, h}+G_{2}^{2, h} \\
& \lambda_{4, h-1}=\lambda_{h}+G_{4}^{1, h}+G_{4}^{2, h} \\
& \sigma_{h-1}=\sigma_{h}+G_{\sigma}^{1, h} \\
& \delta_{h-1}=\delta_{h}+G_{\delta}^{1, h}+G_{\delta}^{2, h} \\
& \frac{Z_{h-1}}{Z_{h}}=1+G_{z}^{1, h}+G_{z}^{2, h}
\end{aligned}
$$

where $(i=2,4)$

a) $G_{i}^{1, h}, G_{\delta}^{1, h}$ and $G_{z}^{1, h}$ depend only on $\lambda_{i, 0}, \delta_{0} ; \ldots \lambda_{i, h}, \delta_{h}$ and are given by series of terms involving only the Mattis model part of the propagator $g_{\omega, L}^{k}(x-y)$, so they coincide with the Mattis model Beta function

b) $G_{\sigma}^{2, h}, G_{i}^{2, h}, G_{\delta}^{2, h}, G_{z}^{2, h}$ are given by a series of terms involving at least a propagator $C_{2, \omega}^{k}(x-y)$ or $g_{\omega,-\omega}^{k}(x-y)$ with $k \geq h$.

By a simple explicit computation

$$
\begin{gathered}
G_{z}^{1, h}=\lambda_{h}^{2}\left[\beta_{2}+\bar{G}_{z}^{h}\right] \\
G_{\sigma}^{1, h}=\lambda_{h} \sigma_{h}\left[\beta_{1}+\bar{G}_{\sigma}^{h}\right]
\end{gathered}
$$

with $\beta_{1}, \beta_{2}>0$ and $\bar{G}_{z}^{h}, \bar{G}_{\sigma}^{h}=O\left(\lambda_{h}\right)$. Moreover $G_{i}^{1, h}, G_{\delta}^{1, h}$ coincide by definition with the Mattis model Beta function, and it was proved in [2],[3] that it is vanishing at any order, i.e.

$$
G_{i}^{1, h}=G_{\delta}^{1, h}=0
$$

Finally as $\left|G_{i}^{2, h}\right|,\left|G_{\delta}^{2, h}\right|,\left|G_{z}^{2, h}\right| \leq K \varepsilon^{2}\left|\sigma_{h}\right| \gamma^{-h}$, one finds, for $h \geq h^{*}$,

$$
\left|\lambda_{i, h-1}-\lambda_{i, 0}\right|<c_{1} \lambda^{2} \quad\left|\delta_{h-1}-\delta_{0}\right| \leq c_{1} \lambda^{2}
$$

and $\sigma_{h} \simeq(\Delta) \gamma^{\eta_{1} h}, Z_{h} \simeq \gamma^{-\eta_{2} h}$ for $h \geq h^{*}$, with $\eta_{1}=-\beta_{1} \lambda+O\left(\lambda^{2}\right), \eta_{2}=\beta_{2} \lambda^{2}+O\left(\lambda^{3}\right)$. As usual in models to which the RG is succesfully applied the flow is essentially described by the second order truncation of the beta function. This shows that it is possible to choose $\lambda$ so small that the conditions of the above lemma are fulfilled. From the definition of $h^{*}$ and the fact that $\sigma_{h} \simeq(\Delta) \gamma^{\eta_{1} h}$ it follows $\sigma_{h^{*}}=\Delta^{1-\beta_{1} \lambda+O\left(\lambda^{2}\right)}$.

As we said the integrations of the $\psi^{\left(<h^{*}\right)}$ (if $h^{*} \geq h_{\beta}$ ) is essentially equivalent to the integration of a single scale $h \geq h^{*}$, so it is well defined by the preceding arguments. If $h^{*}<h_{\beta}$ there is no such integration, and the last scale to be integrated is $h_{\beta}$; from this consideration one obtains easily that the critical temperature is proportional as usual to the gap amplitude.

An expansion for the two points Schwinger function can be derived in a standard way [2] and from the proof of the convergence of the expansion for the effective potential one obtains easily the convergence of the series for the Schwinger function. We can write the r.h.s. of eq.(4) as

$$
\sum_{h=\max \left[h^{*}, h_{\beta}\right]}^{0} \frac{(2 \pi)^{2}}{L \beta} \sum_{\vec{k}^{\prime}} \frac{\sigma_{h}}{Z_{h}} \frac{f_{h}\left(\vec{k}^{\prime}\right)}{k_{0}^{2}+k^{\prime 2}+\sigma_{h}^{2}}\left[1+\lambda \bar{S}_{h}\left(\vec{k}^{\prime}\right)\right]
$$


where $S_{h}\left(\vec{k}^{\prime}\right)$ is a convergent series bounded by a constant; from the above expression one can easily derive the BCS equation eq.(5) as well as the critical temperature.

Note the crucial role of the renormalization of the BCS gap $\sigma_{h}$; it is sensitive to the sign of the interaction and it eliminates or enlarges the singularity of the r.h.s. of eq.(5); neglecting such renormalization one obtains completely different results. In fact our model belongs to the class of universality of the massive Luttinger model, for which it is well known that the bare mass $\Delta$ is renormalized by the interaction to be given by $\Delta^{1-\eta}, \eta=O(\lambda)$; other models belonging to this class are the $X Y Z$ chain or the Yukawa 2 model, see [3],[9]. Note also that no role is plaid in the above analysis by the spin-charge separation; in fact $\left|v_{c}-v_{s}\right|=O(\lambda)$ and such effect is incorporated in the term $\bar{S}^{h}$ in eq.(22)

\section{References}

[1 ] P.W. Anderson The theory of high $T_{c}$ superconductivity, Princeton Press, 1997; and reprints by various authors therein

[2 ] G. Benfatto, G. Gallavotti, A. Procacci, B. Scoppola Comm. Math. Phys 160, 93-171;

[3 ]F. Bonetto, V. Mastropietro, Comm. Math. Phys. 172, 57-93 (1995); Phys. Rev. B 56 1296-1308 (1997); Nucl. Phys. B 497 541-554 (1997); V.Mastropietro, in print on Comm .Math .Phys.; V.Mastropietro, submitted to Math. Phys. Lett.

[4 ] D. Mattis, Physics, 1, 183-193 (1964)

[5 ] A.M. Tsvelik, Quantum field theory in condensed matter physics, Cambridge Un. Press, 1997

[6 ] G.Benfatto, G. Gentile, V.Mastropietro, Jour, Stat. Phys. 92,314, 1998

[7 ] K.Penc, J.Solyom: Phys. Rev. B, 41,1, 704716 (1990); M.Fabrizio, A. Parola, E. Tosatti, 46, 5, 3159-3162 (1992); A.A. Nerseyan, A. Luther, F.V.Kusmartsev, Physics Letters A, 176, 363-370 (1993)

[8 ] Solyom, Advances in Physics, 28, 201-303 (1978) 